\title{
Enhanced piezoelectric shunt design
}

\author{
Chul H. Park ${ }^{\mathrm{a}, *}$ and Daniel J. Inman ${ }^{\mathrm{b}}$ \\ ${ }^{a}$ Department of Mechanical Engineering, Pohang University of Science and Technology, Pohang, KyungBuk, \\ 790-784, Korea \\ ${ }^{\mathrm{b}}$ Center for Intelligent Material Systems and Structures, Department of Mechanical Engineering, Virginia \\ Polytechnic Institute and State University, Blacksburg, VA 24061-0261, USA
}

\begin{abstract}
Piezoceramic material connected to an electronic shunt branch circuit has formed a successful vibration reduction device. One drawback of the conventional electronic shunt circuit is the large inductance required when suppressing low frequency vibration modes. Also, the large internal resistance associated with this large inductance exceeds the optimal design resistance needed for low frequency vibration suppression. To solve this problem, a modified and enhanced piezoelectric shunt circuit is designed and analyzed by using mechanical-electrical analogies to present the physical interpretation. The enhanced shunt circuit developed in this paper is proved to significantly reduce the targeted vibration mode of a cantilever beam, theoretically and experimentally.
\end{abstract}

Keywords: Shunt circuit, vibration damping, piezoceramic, resonant shunt, passive damping

\section{Introduction}

The passive electronic damping is becoming a viable alternative to vibration damping devices due to low cost, lightweight, and good performance. Especially, the inductive shunt damper, which consists of an inductor and resistor, has been used as an effective damping device in many real applications. Forward [1] carried out a preliminary demonstration of the feasibility of using external electronic circuits to damp out mechanical vibrations in optical systems. Hagood and von Flotow [2] showed the analytical models of a resistive (R) and a resonant (LR) electrical shunted piezoelectric with the experimental verifications. Edberg et al. [3] showed that a lightweight electronic circuit could replace the heavy commercial inductor. They also showed the possibility of simultaneously dissipating two modes of vibration by using a piezoelectric material with a tuned shunt circuit. Hollkamp [4] developed a self-tuning piezoelectric vibration absorber which can tune itself to the desired structural mode and track that mode if it varies in frequency. Wu [5] showed a piezo-

*Corresponding author. E-mail: drparkch@postech.ac.kr. ceramic shunt using a piezoelectric element shunted with a parallel resistor and inductor circuit for a passive structural damping and vibration control.

A passive shunt circuit has to be tuned to suppress a target mode like a mechanical vibration absorber. A vibration absorber consists of an attached second mass, spring, and damper, forming a two-degree of freedom system. The second spring-mass and damper system is then tuned to resonate and hence absorb all the vibrational energy of the system. Following the principle of a mechanical absorber, the circuit resonant frequency should be tuned to the mechanical frequency of interest. The resonant circuit consists of three electrical components: a capacitor $\mathrm{C}(\mathrm{PZT})$, an inductor $\mathrm{L}$ and resistor $\mathrm{R}$ as shown in Fig. 1. The two external terminals of the PZT, modeled as a capacitor (since, its electrical property is dominantly capacitive), are connected to the series inductor and resistor shunt circuit. The piezoceramic element is used to convert mechanical energy of a vibrating structure to electrical energy by direct piezoelectric effect. This electric energy is dissipated as Joule heating through the shunt resistor efficiently when the electrical resonant frequency matches the mechanical frequency. At resonance, the reactive components between the inductor and capacitor cancel each 


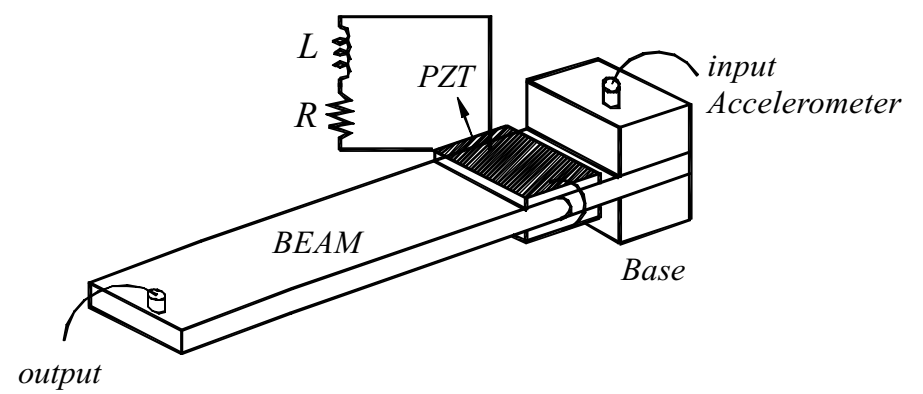

Fig. 1. A schematic drawing of experimental setup with shunt circuit.

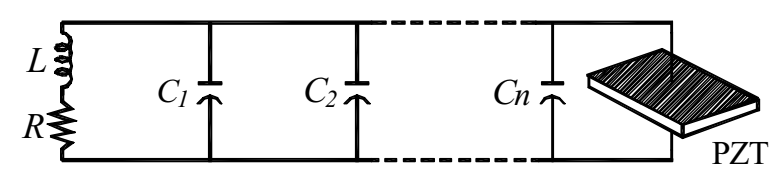

(a)

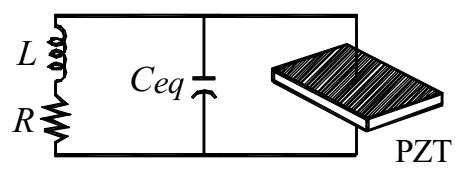

(b)

Fig. 2. Parallel connection of (a) $n$ capacitors and (b) an equivalent circuit.

other and the phase between the current and voltage is zero. As a result, the power factor at resonance becomes one and then, the efficiency of dissipated energy is maximized.

\section{The concept of enhanced shunt circuit (ESC)}

Many researchers have studied to improve the performance of passive shunt damping in last decade. However, there are still several improvements that should be made. One of them is that huge inductances are required to suppress the vibration modes in low frequency range. Though it is possible to generate this required inductance by designing a lightweight synthetic inductor circuit, its large internal resistance in the synthetic inductor circuit to generate a huge inductance, exceeds the optimum resistance value required to suppress a specific vibration mode. To solve this problem, an Enhanced Shunt Circuit (ESC) is presented to be an effective damping device for the structural vibration applications.

The proposed ESC combines an existing shunt circuit with parallel connections of capacitors as shown in Fig. 2. By applying KCL (Kirchhoff's current Law), each current flowing each parallel branch circuit can be [6]

$$
i=i_{P Z T}+i_{2}+i_{3}+\ldots+i_{n}
$$

From the current-voltage relation for PZT and $n$ capacitors,

$$
\begin{aligned}
i & =\left(C_{P Z T}+C_{1}+C_{2}+\ldots+C_{n}\right) \frac{d v}{d t} \\
& =\left(C_{P Z T}+\sum_{n=1}^{N} C_{n}\right) \frac{d v}{d t}
\end{aligned}
$$

The equivalent capacitance $\left(C_{e q}\right)$ is given by:

$$
\begin{aligned}
C_{e q} & =\left(C_{P Z T}+C_{1}+C_{2}+\ldots+C_{n}\right) \\
& =\left(C_{P Z T}+\sum_{n=1}^{N} C_{n}\right)
\end{aligned}
$$

The equivalent capacitance of $n$ parallel capacitors is simply the sum of the individual capacitances including the PZT. If two capacitors $\left(C_{P Z T}\right.$ and $\left.C_{1}\right)$ are connected as parallel,

$$
C_{e q}=\left(C_{P Z T}+C_{1}\right)
$$

The physical interpretation of a shunt circuit that generates additional damping when tuned to a target frequency can be understood when mechanical-electrical analogies are considered. The characteristic equation of a mechanical system and the two solutions are given by:

$$
\begin{aligned}
& s^{2}+\frac{c}{m} s+\frac{k}{m}=0 \\
& s_{1,2}=-\frac{c}{2 m} \pm \sqrt{\left(\frac{c}{2 m}\right)^{2}-\frac{k}{m}}
\end{aligned}
$$

The mechanical natural frequency and damping ratio are defined as follows:

$$
\omega_{m}=\sqrt{\frac{k}{m}}, \quad \zeta_{m}=\frac{c}{2 \sqrt{k m}}
$$


The electric system equation is defined as

$$
L \frac{d i}{d t}+R i+\frac{1}{C} \int i d t=0
$$

The resulting characteristic equation and solution for the eigenvalues are

$$
\begin{aligned}
& L s^{2}+R s+\frac{1}{C}=0 \\
& s_{1,2}=-\frac{R}{2 L} \pm \sqrt{\left(\frac{R}{2 L}\right)^{2}-\frac{1}{L C}}
\end{aligned}
$$

and the electrical resonant frequency and the damping coefficient are

$$
\omega_{e}=\frac{1}{\sqrt{L C}}, \quad \zeta_{e}=\frac{R}{2 \sqrt{L / C}}
$$

Comparing Eq. (5) with Eq. (8), the differential equations for the two systems are of identical form. From Eq. (9), the inductance $L$ (Henry) and capacitance $C$ (Farad) are easily adjusted to obtain a certain electrical resonant frequency. For example, to decrease the required inductance by half, the capacitance should be doubled to obtain the same electrical resonant frequency:

$$
\omega_{e}=\frac{1}{\sqrt{L C}}=\sqrt{\frac{1}{\frac{L}{2} \times 2 C}}
$$

The electric damping ratio is changed as follows:

$$
\zeta_{e}^{\prime}=\frac{R}{2 \sqrt{\frac{L}{2} / 2 C}}=\frac{R}{\sqrt{L / C}}
$$

Comparing Eq. (9) with Eq. (11), the new electrical damping ratio is double the previously calculated damping ratio.

$$
\zeta_{e}^{\prime}=2 \zeta_{e}
$$

This phenomenon will be validated through experimental works in the later section.

A state-space model of the circuit (Fig. 3) can supplement the above formulation to obtain the same electric resonant with half the inductance and double the capacitance. Using a simple application of Kirchhoff's voltage and current law:

$$
\begin{aligned}
& i_{1}=-\left(i_{2}+i_{3}\right) \\
& v=\frac{1}{C_{P Z T}} \int i_{1} d t=\frac{1}{C_{1}} \int i_{2} d t \\
& \quad=\frac{L}{2} \frac{d i_{3}}{d t}+R i_{3} \\
& \int i_{2} d t=-\left(\frac{C_{P Z T} C_{1}}{C_{P Z T}+C_{1}}\right)\left(\frac{1}{C_{P Z T}}\right) \int i_{3} d t .
\end{aligned}
$$

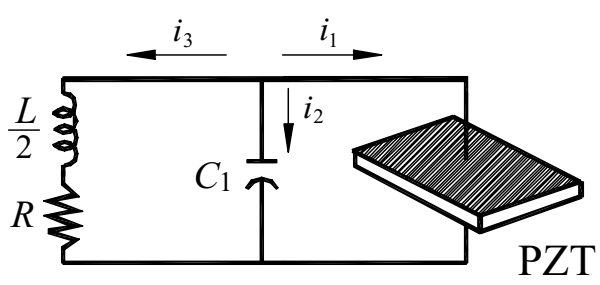

Fig. 3. Shunt circuit with an additional capacitor.

From Eq. (13), the current flowing the series LR branch circuit is

$$
\frac{L}{2} \frac{d i_{3}}{d t}+R i_{3}+\frac{1}{C_{P Z T}+C_{1}} \int i_{3} d t=0
$$

Then by defining the state variables by

$$
x_{1}=\int i_{3} d t, \quad x_{2}=i_{3}
$$

The state space representation of Eq. (14) is

$$
\left[\begin{array}{c}
\dot{x}_{1} \\
\dot{x}_{2}
\end{array}\right]=\left[\begin{array}{cc}
0 & 1 \\
-\frac{1}{\frac{L}{2}\left(C_{P Z T}+C_{1}\right)} & -\frac{R}{L / 2}
\end{array}\right]\left[\begin{array}{l}
x_{1} \\
x_{2}
\end{array}\right]
$$

If $C_{P Z T}=C_{1}$, Eq. (16) becomes

$$
\left[\begin{array}{l}
\dot{x}_{1} \\
\dot{x}_{2}
\end{array}\right]=\left[\begin{array}{cc}
0 & 1 \\
-\frac{1}{\frac{L}{2}\left(2 C_{P Z T}\right)} & -\frac{R}{L / 2}
\end{array}\right]\left[\begin{array}{l}
x_{1} \\
x_{2}
\end{array}\right]
$$

Solving the eigenvalue problem of Eq. (17), also shows that the same electrical frequency is obtained using the half the inductance and double the capacitance that were previously required. This concept will be verified through simulations, experimentally and theoretically.

\section{Theoretical parameters of a resonant shunt}

The piezo shunt circuit generates an additional damping matrix which can be augmented to the equation of motion of a structure system. A pair of piezoelectric actuator/sensor equation [7] is used to derive an additional shunted damping matrix.

$$
\begin{aligned}
& \text { Actuator equation: } \\
& M \ddot{w}+D \dot{w}+K w=f_{\text {ext }}+\theta V_{S H} \\
& \text { Sensor equation: } q=\theta^{T} w+C_{p} V_{S H}
\end{aligned}
$$

where $M, D$, and $K$ are the mass, damping, and stiffness matrices of the piezo/beam system taken at constant field (short circuit). Hence, the system stiffness consists of a base structure stiffness and a short circuit- 
Table 1

Physical and geometrical properties of the beam and piezoceramic

\begin{tabular}{lcccc}
\hline & Young's Modulus $(\mathrm{Pa})$ & Density $\left(\mathrm{kg} / \mathrm{m}^{3}\right)$ & Poisson's ratio $(\nu)$ & Thickness $(\mathrm{mm})$ \\
\hline Beam & $7.1 \mathrm{E} 10$ & 2700 & 0.33 & 1 \\
PZT & $6.3 \mathrm{E} 10$ & 7600 & 0.3 & 0.25 \\
\hline
\end{tabular}

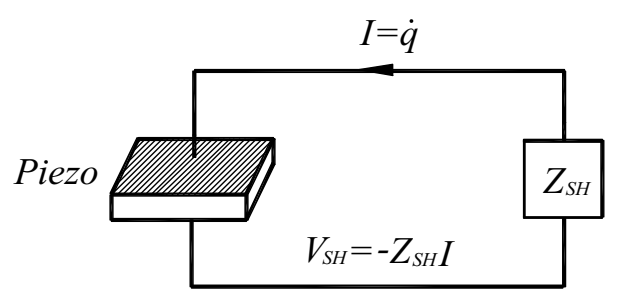

Fig. 4. Feedback current into a PZT due to shunt impedance.

Table 2

Main piezoelectric parameters of G-1195

\begin{tabular}{lcccc}
\hline$d_{31}(\mathrm{~m} / \mathrm{V})$ & $g_{31}(\mathrm{Vm} / \mathrm{N})$ & $K_{3}^{T}$ & $k_{31}$ & $\eta$ (Loss tangent) \\
\hline $180 \mathrm{E}-12$ & $11 \mathrm{E}-3$ & 1800 & 0.35 & 0.02 \\
\hline
\end{tabular}

ed piezoelectric stiffness, that is, $K=K_{s}+K_{p}^{E}$. In the sensor equation, $q$ is the piezoelectric charge matrix and $\theta$ is the electromechanical coupling matrix. This piezoelectric actuator/sensor equation accounts for the effects of dynamic coupling between a structure and an electrical network through the piezoelectric effect. A current equation can be obtained as differentiating the sensor equation (Eq. (19)). Substituting it into the shunt voltage equation as shown in Fig. 4, we can redefine it as follows [8]:

$$
\begin{aligned}
V_{S H} & =-Z_{S H} I \\
& =-Z_{S H}\left(\theta^{T} \dot{w}+C_{p}^{T} \dot{V}_{S H}\right) \\
& =-Z_{S H} \theta^{T} s w-Z_{S H} C_{p} s V_{S H}
\end{aligned}
$$

where $s$ is the Laplace parameter. Therefore, the new defined shunt voltage can be

$$
V_{S H}=\frac{-Z_{S H} \theta^{T} s w}{1+Z_{S H} C_{p} s}
$$

Substituting Eq. (21) into the actuator equation (Eq. (18)), the governing equation of a shunted system can be augmented by adding the shunted damping matrix in the Laplace domain:

$$
\begin{aligned}
& M s^{2} w+\left(C+\frac{Z_{S H} \theta \theta^{T}}{1+Z_{S H} C_{p} s}\right) s w \\
& +K w=f_{\text {ext }}(s) .
\end{aligned}
$$

By dividing the both sides in Eq. (22) into the system stiffness, $K$, we can rewrite Eq. (22) as follows:

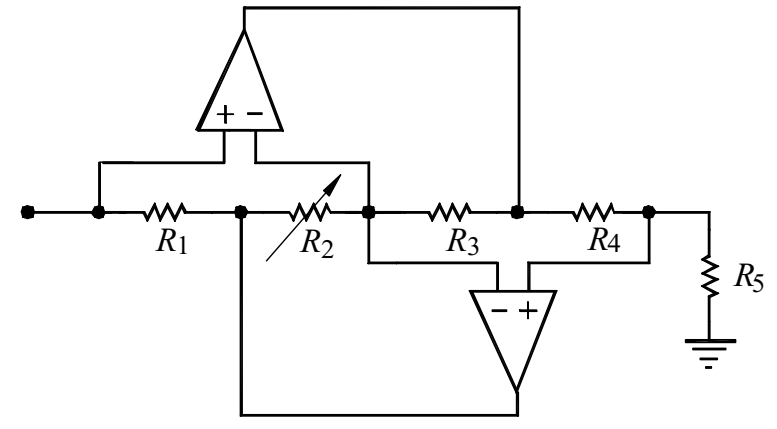

Fig. 5. Circuit diagram of a synthetic inductor.

$$
\begin{aligned}
& \left(\frac{M}{K} s^{2}+\frac{C}{K} s+1+\frac{\theta \theta^{T}}{C_{p} K} \frac{Z_{S H} C_{p} s}{1+Z_{S H} C_{p} s}\right) w(s) \\
& =\frac{f_{\text {ext }}(s)}{K}
\end{aligned}
$$

To obtain a position transfer function of a shunted system, the following parameters are defined.

$$
\begin{aligned}
\omega_{n}^{E} & =\sqrt{\frac{K}{M}}, \quad \gamma=\frac{s}{\omega_{n}^{E}}, \\
\frac{C}{K} s & =2 \xi \gamma, \quad \hat{Z}=Z_{S H} C_{p} s, \\
K_{i j}^{2} & =\frac{\theta \theta^{T}}{C_{p} K}
\end{aligned}
$$

where $\omega_{n}^{E}$ is a natural frequency of a mechanical system with the short circuited piezoelectric material, $\gamma$ is a non-dimensional frequency, and $K_{i j}$ is a generalized electromechanical coupling constant. By using the parameters defined above, Eq. (23) can be rewritten as:

$$
\left(\gamma s^{2}+2 \xi \gamma+1+K_{i j}^{2} \frac{\hat{Z}}{1+\hat{Z}}\right) w=w_{s t}
$$

where $w_{s t}$ is the static displacement of a system. The transfer function of a mechanical structure with the shunted piezoelectric material can be

$$
\frac{w}{w_{s t}}=\frac{1+\hat{Z}}{(1+\hat{Z})\left(\gamma^{2}+2 \xi \gamma+1\right)+K_{31}^{2} \hat{Z}}
$$

In case of an inductance shunt branch circuit, the $\hat{Z}$ can be rearranged by considering $Z_{S H}=L s+R$. 


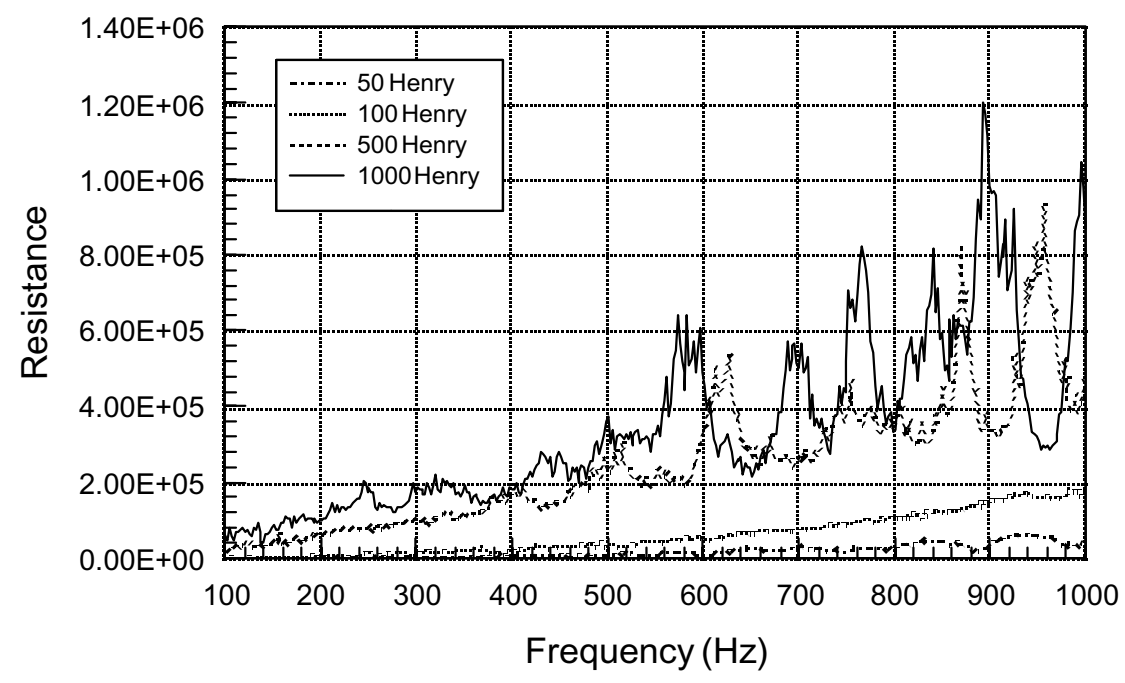

Fig. 6. The inherent resistances of a synthetic inductor.

$$
\begin{aligned}
\hat{Z} & =Z_{S H} C_{p} s=\gamma^{2} L C_{p}\left(\omega_{n}^{E}\right)^{2}+R C_{p} \omega_{n}^{E} \gamma \\
& =\frac{1}{\delta^{2}}\left(\gamma^{2}+r y \delta^{2}\right)
\end{aligned}
$$

where $\delta=\frac{1}{\sqrt{L C_{p} \omega_{n}^{E}}}$ and $r=R C_{p} \omega_{n}^{E}$. Hence, we can write the final form of a system transfer function with an inherent structural damping as follows:

$$
\frac{w}{w_{s t}}=
$$

$$
\frac{\left(\delta^{2}+r \gamma \delta^{2}+\gamma^{2}\right)}{\left(\delta^{2}+r \gamma \delta^{2}+\gamma^{2}\right)\left(\gamma^{2}+2 \xi \gamma+1\right)+K_{31}^{2}\left(\gamma^{2}+r \gamma \delta^{2}\right)}
$$

\section{Experimental description of a resonant shunt}

Several experimental parameters must be determined before an experiment can be performed to verify the theoretical analysis of the Enhanced Shunt Damper proposed in this paper. These parameters include the open and short circuit natural frequencies, the generalized electromechanical coupling coefficient, optimal tuning inductance and capacitance, and the shunting resistance for each targeting mode. The inherent capacitance of the PZT should be determined first by using Eq. (29) below.

$$
C_{p}^{T}=\frac{K_{3}^{T} \times \varepsilon_{0} \times A_{p}}{t_{p}}
$$

where $C_{p}^{T}$ is the capacitance of the PZT at constant stress, $K_{3}^{T}$ is the relative dielectric constant at $1 \mathrm{KHz}$, and the constant $\varepsilon_{0}$ is $8.85 \times 10^{-12} \mathrm{~F} / \mathrm{m}, A_{p}$ is the surface area of PZT and $t_{p}$ is the thickness of the PZT. The product of $K_{3}^{T} \varepsilon_{0}$ is called the permittivity of the dielectric denoted $\varepsilon$. The PZT capacitance at constant strain, $C_{p}^{S}$, is obtained from the following equation which is dependent upon the electromechanical coupling coefficient, $k_{31}$ provided by the manufacturer.

$$
C_{p}^{S}=C_{p}^{T}\left(1-k_{31}^{2}\right)
$$

The generalized electromechanical coupling constant for a piezoelectric element bonded to a structure can be obtained from the frequency change of the electric boundary conditions [2].

$$
K_{31}^{2}=\frac{\left(\omega_{n}^{D}\right)^{2}-\left(\omega_{n}^{E}\right)^{2}}{\left(\omega_{n}^{E}\right)^{2}}
$$

Here, $\omega_{n}^{D}$ and $\omega_{n}^{E}$ are the natural frequencies of the structural mode of interest with an open circuit piezoelectric and a short circuit piezoelectric element, respectively. These frequencies can be obtained from the frequency response function of a mechanical system. The other optimum tuning parameters are calculated from the generalized electromechanical coupling constant [2]:

$$
\delta_{o p t}=\sqrt{1+K_{31}^{2}}, \quad r_{o p t}=\sqrt{2} \frac{K_{31}}{1+K_{31}^{2}}
$$

where $\delta_{o p t}$ is the optimal tuning ratio, $r_{o p t}$ is the electrical damping ratio. The inductance for tuning the targeted mechanical resonant frequency can be found from the electrical resonant frequency.

$$
\omega_{e}=\frac{1}{\sqrt{L C_{p}^{S}}}
$$




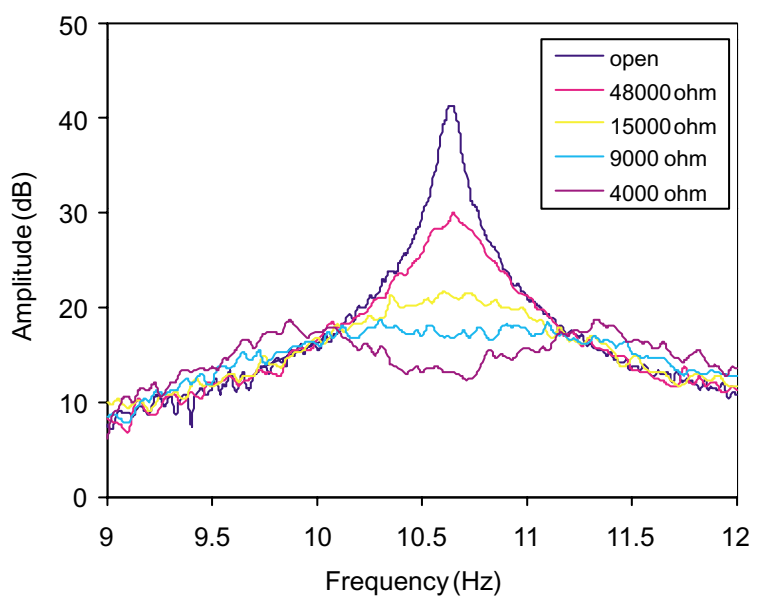

(a)

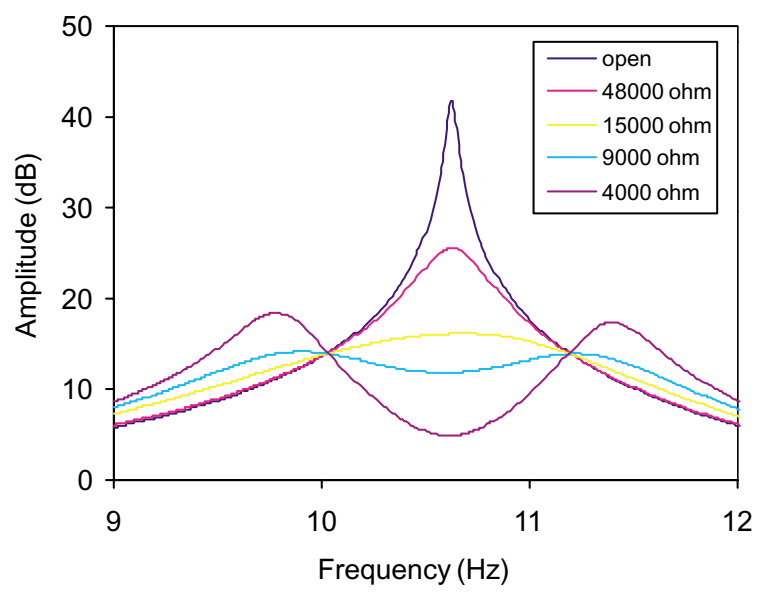

(b)

Fig. 7. Frequency response curve with full inductance $(890 \mathrm{H})$ : (a) experiment and (b) theory.

The experiments with a resonant shunt branch circuit are performed for two different inductance values at the first mode frequency. A pair of $0.063 \mathrm{~m} \times 0.038 \mathrm{~m}$ PZT patches is bonded to each side of the root of the cantilever beam (which is $0.3 \mathrm{~m}$ long and $0.038 \mathrm{~m}$ wide) by using Epoxy Adhesive. The pair of PZT patches is poled through their thickness and elongate lengthwise so that they are operating in transverse mode. The beam is grounded and wired in parallel to produce opposite fields in the top and bottom piezoceramics. This causes a moment on the beam when the top PZT contracts as the bottom one expands. Tables 1 and 2 show the physical and geometrical parameters of the aluminum sheet and piezoceramic material (Type G1195, Piezo Electric Products, Inc).

The internal function generator of the spectrum analyzer (DSP Siglab) is used to generate a random exci- tation input force. This random signal is used to excite a beam which is mounted on an APS shaker (Acoustic Power System Inc.) being driven through a power amplifier (APS Model 114). The input signal is measured by an accelerometer (KISTLER 8630A50) which is attached to the APS shaker. The tip dynamic response (output signal) of the beam is measured by an accelerometer (PCB 309A) and is fed to the spectrum analyzer to determine its frequency content. The magnitude ratio $(\mathrm{dB})$ and the phase shift (degree) of the system response are automatically displayed on the screen. Thus, the transfer function between the input and output can be obtained.

An active filter [9] is used as a synthetic inductor in the shunt branch circuit as shown in Fig. 5. The advantages of this inductor are due to its convenience, lightweight, its ability to generate various inductances. $R_{4}$ is ordinarily a capacitor, with the other impedances being replaced by resistors, creating an inductor $L=R^{*} C$, where $R^{*}=R_{1} R_{2} R_{5} / R_{2}$. By changing the variable resistor $R_{2}$, various inductance values could be obtained. However, the gyrator circuit is not a pure inductor, it creates a resistive component which is not desirable for designing the optimal resistance in the shunt branch circuit. Figure 6 shows the inherent resistances inside a synthetic inductor with various frequencies.

\section{Results and discussions}

The resonant shunt damper is applied to suppress the vibration amplitude of a cantilever beam structure. In Figs 7 and 8 shows the frequency transfer response with different shunt resistances, experimentally and theoretically. The experimental results cover the first bending mode frequency range $(9-12 \mathrm{~Hz})$. Comparisons are shown between the amplitudes of vibration when the shunt circuit is not activated (open circuit) and activated using different resistor values at a fixed room temperature. In addition, tests with full and half inductance were also performed to validate the theoretical analysis.

After measuring the frequency response function between output and input voltage, the open and short circuit frequencies measured are $10.625 \mathrm{~Hz}$ and $10.5 \mathrm{~Hz}$ for the first mode. Using Eq. (31), the generalized electro-mechanical coupling coefficients calculated for the PZT patches are 0.15 for the first mode frequency. The electrical resonant frequency equation (Eq. (33)) is used in order to calculate the inductance in shunt 


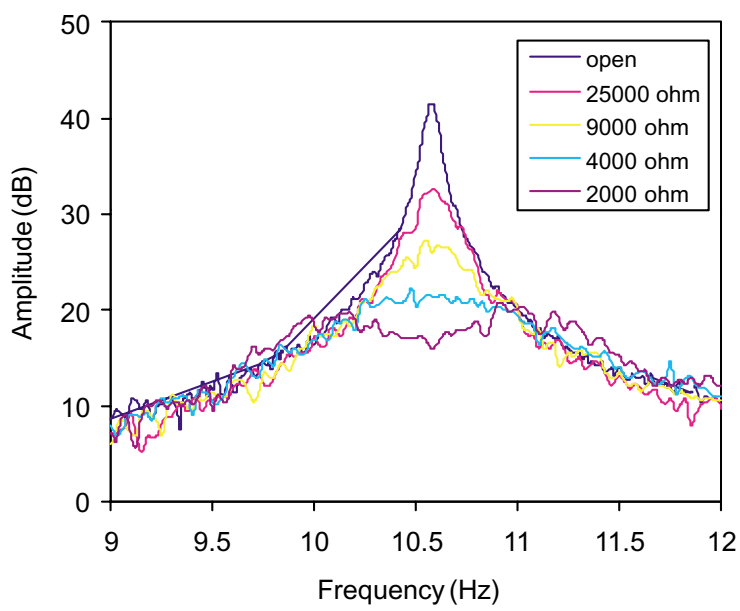

(a)

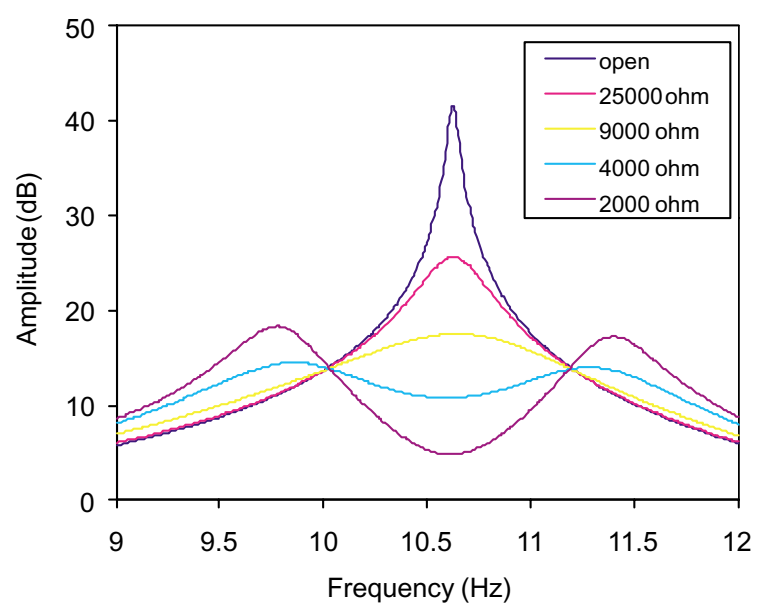

(b)

Fig. 8. Frequency response curve with half inductance $(445 \mathrm{H})$ : (a) experiment and (b) theory.

branch circuit. By the calculation, 890 Henry was required to control the first mode vibration amplitude. This amount of inductance is difficult to obtain from the commercial manufacturers.

From Figs 7 and 8, it is evident that decreasing resistance results in improving the vibration attenuation characteristics of the first bending mode. When the resistance is decreased below the optimal resistance, the two peaks rise up, which is a similar phenomenon of mechanical absorber damping. From the Fig. 7, the shunted piezoelectric pair is found to produce about $25 \mathrm{~dB}$ vibration amplitude reduction from the open circuit peak vibration amplitude. The experimental and theoretical effects of the shunt piezo damper with half inductance (445 Henry) and double conductance are shown in Fig. 8. In this case, only half resistance is needed to obtain the same vibration amplitude reduction. This phenomenon was explained by the analysis given in Eq. (12). One more thing should be noted that the theoretical transfer response curves in Figs 7 and 8 below optimal resistance are slightly different from experimental results. This fact can be due to the inherent resistance of the synthetic inductor discussed in the previous section.

\section{Conclusions}

In this paper, a technique that is capable of reducing the structure vibration amplitude using the electrical passive damper with a half, a quarter, or even onetenth the previously required inductance is introduced. To verify the effectiveness of this technique, a theory was developed with the consideration of mechanicalelectrical analogies. Also, this technique was experimentally tested with a cantilever beam. The experiments demonstrated the successful vibration amplitude reductions with a full and a half inductance at the first bending mode. The vibration amplitude was decreased about $25 \mathrm{~dB}$ at the first mode frequency. When the shunt circuit with a half inductance was used, the electric damping was doubled. Hence, the developed technique presents an economical damping tool for suppressing the structural vibration amplitude.

\section{References}

[1] R.L. Forward, Electronic Damping of Vibrations in Optical Structures, Applied Optics 18(5) (1979), 690-697.

[2] N.W. Hagood and A. von Flotow, Damping of Structural Vibrations with Piezoelectric Materials and Passive Electrical Networks, J. of Sound and Vibration 146(2) (1991), 243-268.

[3] D. Edberg, A. Bicos and J. Fechter, On Piezoelectric Energy Conversion for Electronic Passive Damping Enhancement, Proceedings of Damping, WL-TR-91-3078 2(1991), GBA1-10.

[4] J. Hollkamp and T. Starchville, Jr., A Self-Tuning Piezoelectric Vibration Absorber, J. of Intelligent Material Systems and Structures 5 (1994), 559-566.

[5] S. Wu, Piezoelectric Shunts with a Parallel R-L Circuits for Structural Damping and Vibration Control, Proc. of SPIE 2720 (1996), 259-269.

[6] D. Johnson, J. Hilburn and J. Johnson, Basic Electric Circuit Analysis, Prentice-Hall, Inc., 1978.

[7] N. Hagood, W. Chung and A. von Flotow, Modeling of Piezoelectric Actuator Dynamics for Active Structural Control, J. of Intelligent Material Systems and Structures 1 (1990), 327-354.

[8] C.H. Park and D.J. Inman, A Uniform Model for Series R-L and Parallel R-L Shunt Circuits and Power Consumption, Proc. of SPIE 3668 (1999), 797-804.

[9] P. Horowitz and W. Hill, The Art of Electronics, Cambridge University Press, 1989. 

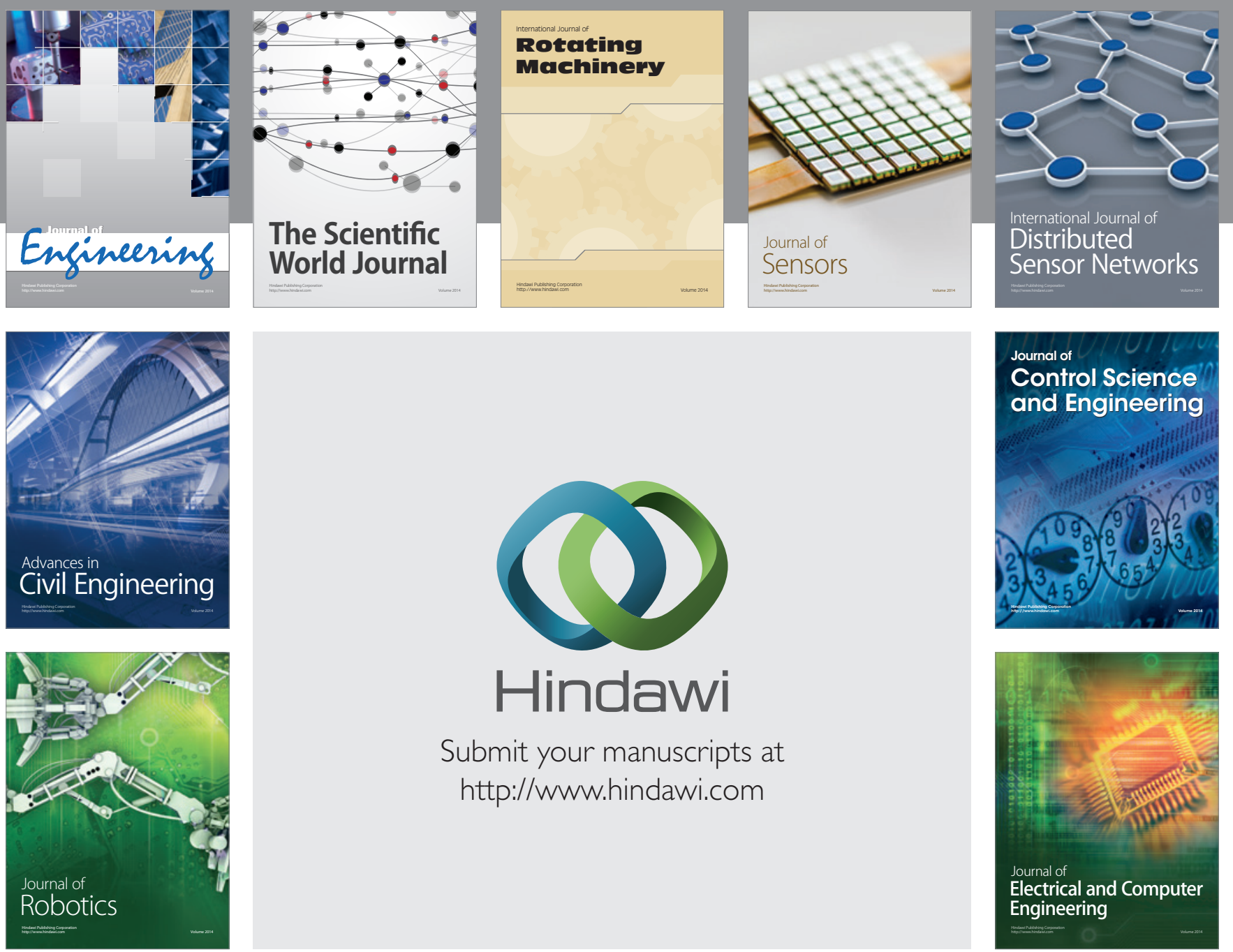

Submit your manuscripts at

http://www.hindawi.com
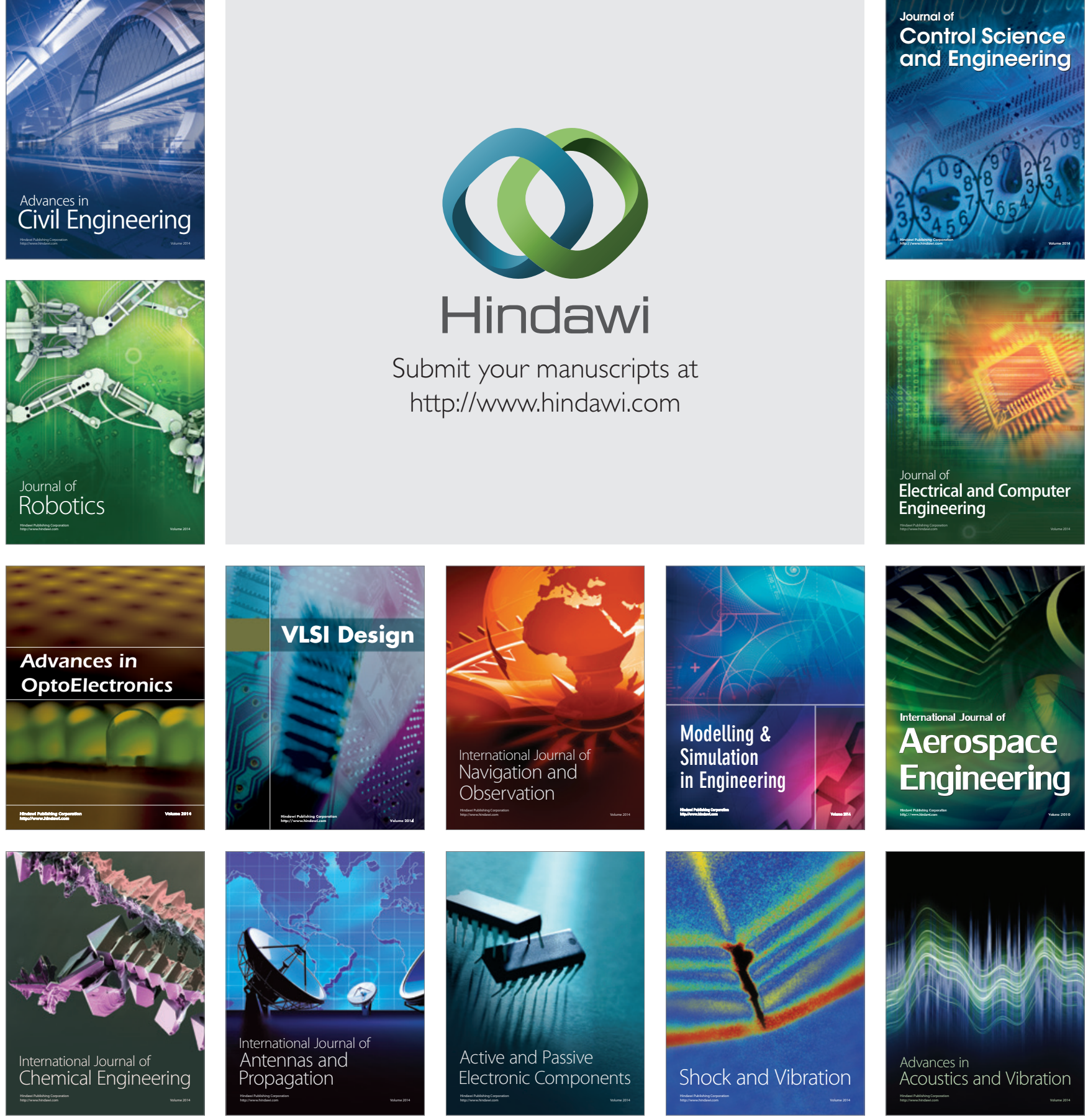